\title{
Overview of marine Controlled-Source Electromagnetic Interferometry by multidimensional deconvolution
}

\author{
Jürg Hunziker, Evert Slob and Kees Wapenaar
}

\section{summary}

Interferometry by multidimensional deconvolution for marine Controlled-Source Electromagnetics can suppress the direct field and the airwave in order to increase the detectability of the reservoir. For monitoring, interferometry by multidimensional deconvolution can increase the source repeatability. We give an overview over the method and discuss a possible path of research for the future. 


\section{Amsterdam '14}

\section{Introduction}

Seismic interferometry is a method broadly applied in seismics for various purposes like extracting a signal from noise (e.g., Draganov et al., 2006) or imaging below a complex overburden (e.g., van der Neut et al., 2011). Schuster (2009) or Wapenaar et al. (2011) give an overview of seismic interferometry. However, in Controlled-Source Electromagnetics (CSEM) this concept is hardly ever used although it could bring some benefits as well. In marine CSEM, which is the focus of this abstract, the response of a subsurface reservoir is covered at short offsets by the direct field and at large offsets, in case of a shallow-sea situation, by the airwave (Amundsen et al., 2006). In this case, interferometry can increase the detectability of the reservoir by suppressing the direct field and the airwave (Hunziker et al., 2013). In a monitoring case or if the data are inverted for the subsurface conductivity distribution, interferometry can decrease the source uncertainty (Hunziker et al., 2011). In this abstract, we give an overview of interferometry for marine CSEM and discuss a possible path of research for the future.

\section{Method}

In seismics, several types of interferometry are used. Probably the most applied type of interferometry is by cross-correlation. Because interferometry by cross-correlation assumes the medium to be lossless, it is not applicable to the diffusive electromagnetic fields of CSEM. Instead, we use interferometry by multidimensional deconvolution (MDD), because it does not assume a lossless medium.

Interferometry by MDD for marine CSEM consists of three major steps. Firstly, the synthetic aperture source concept (Fan et al., 2010) is applied to filter out the high-wavenumber content that is aliased because of the typically large receiver spacing. The synthetic aperture source concept combines many source positions to create one big source exploiting the dense sampling on the source side of marine CSEM. By choosing the length of the source well, all the aliased high-wavenumbers are suppressed (Hunziker et al., 2012). In the second step, the electromagnetic field is decomposed into downward decaying and upward decaying fields. For this, we use an algorithm (Slob, 2009) that requires the four horizontal components of the electromagnetic field to be measured on a $2 \mathrm{D}$ receiver-grid. This implementation assumes further that the medium parameters at the receiver level are known and laterally constant. In the third step, the upward decaying field is deconvolved by the downward decaying field retrieving the reflection response. Because the decomposition algorithm also separates the transverse magnetic (TM) and the transverse electric (TE) modes, the TM- and TE-mode reflection responses can be retrieved by deconvolving the corresponding decomposed fields. The name-giving deconvolution can either be carried out in the wavenumber domain or in the space domain (Hunziker et al., 2013). Performing the deconvolution in the wavenumber domain is much more efficient, because the actual deconvolution is reduced to an elementwise division, but it assumes the medium to be laterally invariant everywhere.

Because this method is a data-driven method, properly sampled data are required. Properly sampled means in this case, that the grid has to be regular or can be regularized and that there should not be any gaps in the data. Thus, also the often clipped amplitudes around zero offset need to be available. If that is not the case, they might be replaced by a numerical simulation of the direct field in the water.

\section{Results}

In order to investigate if applying interferometry by MDD increases the detectability of a reservoir, we computed the normalized amplitude for the inline electric field and for the TM-mode reflection response retrieved by interferometry by MDD for a simple layered Earth model (data taken from Hunziker et al., 2013). The normalized amplitude is a division of a dataset with reservoir by a dataset without reservoir. Normalized amplitudes different from 1 indicate that the reservoir can be detected. The normalized amplitude of the inline electric field is only slightly larger than 1, except for a few datapoints (Figure 1a). Because these datapoints lie close to zero-crossings of the electric field, they are considered to be 


\section{Amsterdam '14}

numerical artifacts from datapoints that have blown up in the division. In contrast, the normalized amplitude for the TM-mode reflection response is larger than 2 almost everywhere (Figure 1b). Consequently, retrieving the reflection response using interferometry increases the reservoir detectability.
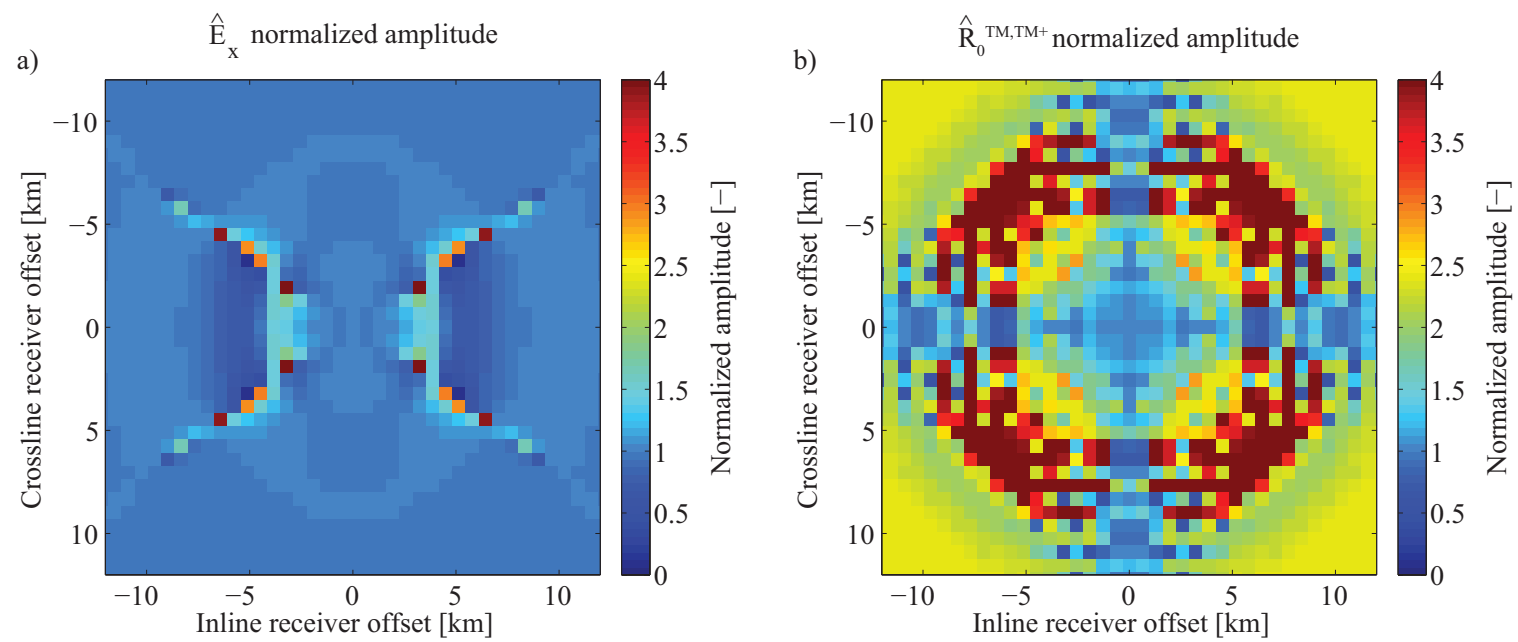

Figure 1 Reservoir detectability for a) the inline oriented electric field and for $b$ ) the reflection response retrieved by interferometry by MDD (modified from Hunziker et al. (2013)).

\section{Conclusions and Outlook}

We have shown that interferometry applied to marine CSEM data increases the reservoir detectability and the source repeatability. Because interferometry poses quite some requirements on the data, an acquisition fulfilling this setup might be quite expensive. The question is thus, if the benefits justify the costs also after an inversion for the subsurface conductivity distribution has been carried out. Therefore, the next step of research should quantify the improvement of the retrieved subsurface conductivity distribution if the reflection response is inverted instead the full electromagnetic fields.

\section{Acknowledgements}

This research is supported by the The Netherlands Research Centre for Integrated Solid Earth Sciences (ISES).

\section{References}

Amundsen, L., Løseth, L., Mittet, R., Ellingsrud, S. and Ursin, B. [2006] Decomposition of electromagnetic fields into upgoing and downgoing components. Geophysics, 71, G211-G223, doi:10.1190/1.2245468.

Draganov, D., Wapenaar, K. and Thorbecke, J. [2006] Seismic interferometry: Reconstructing the earth's reflection response. Geophysics, 71, SI61-SI70, doi:10.1190/1.2209947.

Fan, Y. et al. [2010] Synthetic aperture controlled source electromagnetics. Geophys. Res. Lett., 37, L13305.

Hunziker, J., Slob, E., Fan, Y., Snieder, R. and Wapenaar, K. [2012] Two-dimensional controlled-source electromagnetic interferometry by multidimensional deconvolution: spatial sampling aspects. Geophysical Prospecting, 60, 974-994.

Hunziker, J., Slob, E., Fan, Y., Snieder, R. and Wapenaar, K. [2013] Electromagnetic interferometry in wavenumber and space domains in a layered earth. Geophysics, 78(3), E137-E148, doi:10.1190/geo2011-0510.1.

Hunziker, J., Slob, E., Wapenaar, K., Fan, Y. and Snieder, R. [2011] Time-lapse controlled-source electromagnetics using interferometry. The Leading Edge, 30(5), 564-567, doi:10.1190/1.3589112.

Schuster, G. [2009] Seismic Interferometry. Cambridge University Press.

Slob, E. [2009] Interferometry by deconvolution of multicomponent multioffset GPR data. IEEE Transactions on Geoscience and Remote Sensing, 47, 828-838.

van der Neut, J., Thorbecke, J., Mehta, K., Slob, E. and Wapenaar, K. [2011] Controlled-source interferometric redatuming by crosscorrelation and multidimensional deconvolution in elastic media. Geophysics, 76(4), SA63-SA76, doi:10.1190/1.3580633.

Wapenaar, K. et al. [2011] Seismic interferometry by crosscorrelation and by multi-dimensional deconvolution: a systematic comparison. Geophysical Journal International, 185, 1335-1364. 